\title{
Choix d'ouvrages traitant de la critique et des méthodes historiques avec des notes critiques et historiques
}

\section{Marie-Claire Daveluy}

Volume 17, numéro 3, décembre 1963

URI : https://id.erudit.org/iderudit/302307ar

DOI : https://doi.org/10.7202/302307ar

Aller au sommaire du numéro

Éditeur(s)

Institut d'histoire de l'Amérique française

ISSN

0035-2357 (imprimé)

1492-1383 (numérique)

Découvrir la revue

Citer ce document

Daveluy, M.-C. (1963). Choix d'ouvrages traitant de la critique et des méthodes

historiques avec des notes critiques et historiques. Revue d'histoire de

l'Amérique française, 17(3), 457-463. https://doi.org/10.7202/302307ar 


\section{BIBLIOGRAPHIE}

\section{CHOIX D'OUVRAGES TRAITANT DE LA CRITIQUE ET DES MÉTHODES HISTORIQUES AVEC DES NOTES CRITIQUES ET HISTORIQUES}

\section{ORDRE CHRONOLOGIQUE DES ÉDITIONS ORIGINALES}

$$
\text { - } 1842 \text { (?) - }
$$

1. L'abbé Jacques BALMÈs, 1810-1848, L'Art/ d'arriver au vrai/. Traduit de l'espagnol par Edouard Manec. 15e édition. Paris, 1903.

Note. Un des auteurs les plus érudits de son temps. Un thomiste ardent. Il fut un des admirateurs de l'œuvre de Chateaubriand: Le Génie du christianisme. L'abbé Balmès connut la grande notoriété par son ouvrage: Le protestantisme comparé au catholicisme. Nos bibliothèques, en général, possèdent l'Art d'arriver au vrai, où sont formulés des principes qui ne vieilliront jamais complètement. Cet ecclésiastique mourut à 38 ans. L'édition originale fut publiée vers 1842. Le titre espagnol se présente sous deux façons différentes: El criterio, ou bien: El arte pensefor.

$$
-1883-
$$

2. Le Père Charles DE SMEDT, s.j., 1833-1911, Principes/ de la/ critique historique/ par le/P. Ch. de Smedt, s.j./, bollandiste./ Liège/ Librairie de la Société bibliographique belge/ 2, rue Nagelmakers, 2/. Paris, Librairie de la Société bibliographique/ 195, boulevard Saint-Germain/ [et] Société générale de la Librairie catholique/ 76 rue des Saints-Pères/ [1883]. 292 pages. $18.5 \times 12.5 \mathrm{~cm}$. Edition originale.

Note. Ce jésuite, d'origine belge, fut affecté très jeune aux travaux de la célèbre Ecole des Bollandistes fondée en 1642 par Jean Bolland. Il renouvela entièrement la méthodologie de 
cette institution en publiant l'ouvrage ci-dessus décrit. Il parut à tous, à l'époque, "presque révolutionnaire". Il inaugura ce que l'on appelle souvent le néo-bollandisme. Quelques bibliothèques montréalaises comptent cet ouvrage indispensable à tout historien.

3. Jean MOELlER, 1792-1864, professeur d'histoire générale à l'Université catholique de Louvain et l'un des fondateurs de cette institution en 1834, Traité des études historiques. [Réimpression de l'édition originale de 1887]. Ouvrage posthume publié avec des additions par son fils Charles Moeller, professeur à la même Université de Louvain. Paris, Ernest Thorin [... ], 7, rue de Médicis, 1892.2 vol. $25 \mathrm{~cm}$.

Note. Les Moeller, des Belges, demeurent une famille de philosophes, de savants et d'écrivains depuis plusieurs générations. L'ancêtre Nikolaï, était un Norvégien, protestant converti. Un des derniers représentants de la famille, l'abbé Charles Moeller, petit-fils de Jean et neveu de Charles, est le fils de Nicolas, médecin et écrivain. L'abbé Moeller, professeur à son tour à l'Université de Louvain, vient de publier (de 1952 à 1956), trois volumes de son magnifique ouvrage intitulé: Littérature $d u X X e$ siècle et christianisme. L'œuvre de Jean et Charles Moeller que nous venons de décrire garde toujours sa valeur formatrice.

4. Charles-Victor LANGLoIS 1863-1929 et Charles SEIgNoBos, 1854-1942, Introduction aux études historiques. Paris, Hachette, 1898. XVIII-308 pages. In-12. Edition originale.

Note. La quatrième édition porte le millésime de 1909. Nous l'avons vainement cherchée dans les nombreuses librairies parisiennes, lors de notre séjour en France en 1949. Cet ouvrage rarissime méritait pourtant d'être réédité. Le travail de MM. Langlois et Seignobos demeure indispensable aux débutants dans le domaine de l'histoire. C'est sans cesse le ton du professeur s'exprimant avec simplicité, clarté et conviction. Se souvenir cependant que ces deux savants auteurs sont des rationalistes, ce qui se voit du reste quand il s'agit par exemple de tenir compte (ou non) du miracle en histoire; ou encore, d'une philosophie de l'histoire à base de croyances chrétiennes. Cette remarque restrictive ne doit jamais être oubliée et constitue une mise en garde sérieuse. Mais une fois averti des idées positivistes dangereuses des auteurs, il est difficile d'ignorer la métho- 
dologie préconisée par ces maîtres. (Lecture recommandée pour réfuter les assertions positivistes des MM. Langlois et Seignobos: l'ouvrage des Pères Garraghan et Delanglez, jésuites, dont nous parlerons bientôt dans ses pages).

Note supplémentaire. La dernière lettre de Charles Seignobos à Ferd. Lot, sur la méthodologie historique (voir La Revue historique, 77e année, t. CCX, juillet-septembre 1953. Paris, Presses universitaires de France).

En 1941, Charles Seignobos écrivit à Ferdinand Lot dont il venait de relire l'ouvrage magnifique: Les Invasions barbares et le peuplement de l'Europe, une lettre importante sur les principes de la méthode historique, matière autour de laquelle il avait réfléchi toute sa vie. A la mort de Ferdinand Lot en 1953 ce document fut retrouvé parmi les papiers du maîtrehistorien. Il parut peu après. Travail profitable aux méthodologistes de l'histoire. Charles Seignobos y approfondit les principes de la critique et leur donne certaines formules définitives.

Nouvelle note supplémentaire sur les éditions de cet ouvrage qui a compté d'innombrables lecteurs. Edition originale, 1898. Il $\mathrm{y}$ eut trois réimpressions de cette édition: 1899 (2e éd.); 1905 (3e éd.) ; 1909 (4e éd.).

5. Georges DESDEVISES DU DÉZERT et Louis BRÉHIER, membre de l'Institut, 1868-1952, Le Travail historique. Paris, Librairie Bloud \& Cie, 4 rue Madame, 4, 1908. 82 pages. $18 \mathrm{x}$ $11.5 \mathrm{~cm}$. (Collection Science et Religion - Questions historiques). Edition originale.

Note. Cette brochure peut être utile à l'occasion. Elle est d'une lecture attachante, avec ses conseils judicieux. L'on fera bien de lire et relire le chapitre V et dernier intitulé: La Composition et le style. Comme la plupart de nos bibliothèques possèdent la savante collection Science et Religion où plus de sept cents sujets sont abordés par des maîtres, il sera facile de jeter un coup d'œil sur l'œuvre ci-dessus.

6. Léopold FoNCK, Le travail scientifique. Ecole-pratique, adapté de l'allemand par J. Bourg et A. Decisier. Paris, Beauchesne, 1911.

Note. Ouvrage irremplaçable, au témoignage d'un historien, qui a pu en tirer grand profit au début de sa carrière. 
7. Ecole nationale des Chartes, Livre du Centenaire (18211921), [par Maurice Prou, membre de l'Académie des inscriptions et belles-lettres, directeur de l'Ecole des Chartes]. 2 vol., Paris, Auguste Picard, éditeur, 82, rue Bonaparte, 82, 1921. $18 \times 12 \mathrm{~cm}$.

Note. Que de connaissance on acquiert sur l'enseignement de l'histoire en lisant ces deux petits volumes de près de 400 pages chacun. Le but de l'Ecole nous fait connaître l'objet des cours qu'on y donne: "former des érudits capables de classer des archives, de diriger les bibliothèques, de publier avec critiques, les documents et les manuscrits, et même de composer des ouvrages d'histoire".

Comment ne pas louer, puisque l'occasion est propice, la revue propre de l'Ecole des Chartes: la Bibliothèque de l'Ecole des Chartes, créée dans la seconde moitié du XIXe siècle. Elle a contribué - et elle contribue encore - par la sévérité de ses jugements à maintenir et même à relever le niveau des études historiques [...] On a écrit de cette admirable revue "qu'elle donne le ton à toute la littérature historique".

8. Paul FouRNIER [chartiste], 1853-?, Conseils pratiques pour le classement et l'inventaire des Archives et l'édition des documents historiques écrits. Paris, Champion, 1924. IV92 p. In-8.

Note. Indispensable à consulter.

$$
-1926-
$$

9. Semaine d'histoire du Canada, Montréal, 1925. Première Session tenue à la Bibliothèque Saint-Sulpice, à Montréal, du 23 au 27 novembre 1925, sous les auspices de la Société historique de Montréal. Compte rendu et Mémoires. [Introduction par Aegidius Fauteux, secrétaire]. Montréal, Société historique de Montréal, 1926. 456 pages. $22.5 \mathrm{~cm} \mathrm{x}$ $16 \mathrm{~cm}$. Edition originale.

Note. Nos étudiants, dans le domaine de l'histoire, se doivent de lire avec attention cet ouvrage. Je leur recommande en particulier les travaux du R. P. A.-M. Mignault, o.p., de l'abbé H.-A. Scott, de M. P.-G. Roy, de M. Gustave Lanctôt, de quelques autres encore, soucieux de saine et sûre méthodologie. 
10. Dom Henri Quentin, moine bénédictin de l'abbaye de Solesmes, France, Essais de critique textuelle (Ecdotique). Paris, Editions Auguste Picard, 82, rue Bonaparte, 82, 1926. 177 pages. $22.5 \mathrm{~cm}$. x $14.5 \mathrm{~cm}$. Edition originale. Note. Ouvrage d'érudition autour des textes bibliques tout particulièrement. Mais il y a des principes généraux de critique textuelle qui sont à méditer et à suivre.

- 1927-1928 -

11. Histoire et Historiens depuis cinquante ans. Méthodes, organisation et résultats du travail historique de 1876 à 1926. Recueil publié à l'occasion du cinquantenaire de la Revue historique, avec la collaboration de nombreux érudits et historiens [parmi lesquels nous citerons: MM. Chs Bémont, H. P. Biggar (un Canadien), Louis Bréhier, Gustave Cohen, Augustin Fliche, René Grousset, Louis Halphen, H. Maspero, Henri Pirenne]. Paris, Librairie Félix Alca, 108, boulevard Saint-Germain, 108, 1926-1927. 2 vol. (vol. I: 470 pages; vol. II: de 471 à 758 pages). $25 \mathrm{~cm}$. (Bibliothèque de la Revue Historique). Edition originale.

Note. Nous n'avons pas à souligner l'importance d'un tel ouvrage. L'historiographie de quatre continents nous est présentée sous tous leurs aspects respectifs. Les méthodes historiques ont grandement progressé depuis 1876. On s'en convainc en lisant ce recueil. Mais au Canada, à part de brillantes exceptions, la formation historique laisse vraiment à désirer. Des cours se donnent heureusement depuis quelques années sur la méthodologie de l'histoire. M. Gustave Lanctot fut le premier à donner de ces cours. Il professait alors à l'Université d'Ottawa. Nous devons aussi louer l'initiative d'un de nos maîtres en histoire, M. Guy Frégault (aujourd'hui sous-ministre au Ministère des Affaires culturelles de la province de Québec) qui a enseigné, lui aussi, la méthodologie historique à l'Université de Montréal.

$$
-1933-
$$

12. Paul Harsin, Comment on écrit l'histoire. Liège, 1933. Edition originale.

Note. Cinq éditions (la dernière en 1949) ont paru de cet excellent petit ouvrage. $-1935-$

13. Joseph-Louis-Antoine CALMETte, 1873-1952. Membre de l'Institut, Le Monde féodal. Avant-propos de S[ébastien] 
Charlety, membre de l'Institut, recteur de l'Université de Paris (vers 1935). Paris, les Presses universitaires de France [1935] 490 pages. $20 \times 14.5 \mathrm{~cm}$. (Collection Clio; introduction aux études historiques [no 4$]$ ). Edition originale. Une 2e édition, revue, a paru en 1937.

Note. C'est un admirable manuel d'histoire se spécialisant sur les institutions du Moyen Age. Vraiment, on peut qualifier cet ouvrage de chef-d'œuvre du genre. Science, érudition, rédaction concise et agréable, riches bibliographies générales ou particulières à la fin des chapitres. L'appareil critique ne néglige aucune de ces attributions et tout y est d'une qualité supérieure. Nous recommandons beaucoup cet ouvrage essentiellement formateur en histoire. L'introduction contient avec l'histoire de l'Histoire, des principes et des conseils que nos étudiants auront soin de suivre. Ils se familiariseront ainsi avec les documentssources, manuscrits et imprimés. Ils s'intéresseront du reste avec le sujet que traite l'auteur, car au Canada, sous le régime français, le système féodal formait la structure politique de la colonie.

$-1946-$

14. Gilbert J. GarRaghan, S.J., 1871-1942, Late Research Professor of History, Loyola University, Chicago, A Guide to Historical Method. Edited by Jean Delanglez, S.J., 18911949, Research Professor of History, Loyola University, Chicago (Collection Fordham University Press Book). [Third printing of the first edition published in 1946]. Chicago, The Declan McMullen Company Inc., Distributors, 1951. 482 p. $24 \times 16 \mathrm{~cm}$.

[Inclus dans le même ouvrage]: Bibliographical Citation in the Social Sciences, $A$ Handbook of Style, by Livia Appel, Managing Editor, University of Wisconsin Press [Troisième réimpression de l'édition originale publiée en 1940]. Madison, The University of Wisconsin Press, 1948. 30 pages. $24 \times 16 \mathrm{~cm}$.

Note. Manuel en langue anglaise préparé par des maîtres. Les étudiants bilingues ne sauront que bénéficier des principes de critique préconisés dans cet ouvrage de ces deux regrettés Jésuites, professeurs et historiens. Cependant, il ne faudra pas tenir compte des parties - par exemple ce qui a trait à la ponctuation -, que n'accepteraient point les grammairiens de langue française.

15. Louis-Sigismond-Isaac HALPHEN, 1880-1950, membre de l'Institut, professeur à la Sorbone. Introduction à l'histoire. 
Deuxième édition revue et corrigée. Paris, Les Presses universitaires de France, 108, boulevard Saint-Germain, 108, 1948. 88 pages. $20 \times 15 \mathrm{~cm}$. Edition originale, 1946 .

Note. Excellente étude du créateur et directeur de l'admirable collection Peuples et Civilisation conjointement avec Philippe Sagnac. M. Halphen écrivit le vol. 5 de la collection: Les Barbares des grandes invasions aux conquêtes turques du XIe siècle; aussi, le vol. 6, tout en donnant sa collaboration au 7e sur la Fin du Moyen Age. Ne mettons-nous pas ainsi en évidence la science peu commune de ce professeur formé par la célèbre Ecole des Chartes, en France ? En appendice, deux études prennent place ayant comme titres la première: Les Etapes de la science historique; la seconde: Les Etudes de méthodologie historique.

(à suivre)

Marie-Claire DaveluY. de l'Académie canadienne-française.

\section{Ouvrages reçus et dont un compte rendu paraîtra dans le prochain numéro}

The Hudson's Bay Record Society, vol. XXIV, Northern Quebec and Labrador Journals and Correspondence 1819-35.

W. L. MoRTON, The Kingdom of Canada.

ROBERT V. HINE, Edward Kern and American Expansion.

WALTER ShePPE, First Man West - Alexander Mackenzie's Account of His Expedition Across North America to the Pacific in 1793.

HENRI DORION, La frontière Québec-Terreneuve.

Ge0. BouchaRd, Show-Down ou Cartes sur Table ou Notre bilan après deux siècles d'agitation.

Léo-Paul Desrosiers, Dans le nid d'Aiglons, la Colombe.

COLETTE-ALBERT-SAMUEL, Bibliographie annuelle de l'Histoire de France, Année 1962.

Winston DE VILLE, Marriage Contracts of Natchitoches, 17391803.

Recherches sociographiques, III no 3 (1962); IV, no 1 (1963). Rapports d'archives: Documentos ineditos para la Historia de Colombia - Juan Friede De Orden de La Academia Colombiana de Historia, VIII : 1545-1547.

Rivista Storica Italiana - Anno LXXV - Fasciolo II.

Revista de Historia de América - numéro 51 - Junio De 1961. Instituto Panamericano de Geografia E Historia. 